\title{
Treatment of Thoracic Spine Tuberculosis by Paraspinal Muscle Gap Approach
}

\author{
Jinpeng Zheng1, Shuan Liu², Bing $\mathrm{Hu}^{2 *}$, Jinjun $\mathrm{Li}^{1 *}$ \\ ${ }^{1}$ Medical College, Wuhan University of Science and Technology, Wuhan, China \\ ${ }^{2}$ Department of Orthopaedics, Tianyou Hospital, Wuhan University of Science and Technology, Wuhan, China \\ Email: 512461474@qq.com,897138981@qq.com,675812416@qq.com, ‘hubinghuda@163.com
}

How to cite this paper: Zheng, J.P., Liu, S.A., Hu, B. and Li, J.J. (2017) Treatment of Thoracic Spine Tuberculosis by Paraspinal Muscle Gap Approach. International Journal of Clinical Medicine, 8, 639-651. https://doi.org/10.4236/ijcm.2017.812060

Received: October 28, 2017

Accepted: December 2, 2017

Published: December 5, 2017

Copyright ( 92017 by authors and Scientific Research Publishing Inc. This work is licensed under the Creative Commons Attribution International License (CC BY 4.0).

http://creativecommons.org/licenses/by/4.0/

(c) (i) Open Access

\begin{abstract}
Objective: To evaluate the clinical feasibility and efficacy in treatment of thoracic tuberculosis via paraspinal approach. Methods: From June 2011 to August 2016, 24 patients with mono-segmental thoracic spine tuberculosis were treated by transfacet debridement combined with bone grafting and internal fixation through paraspinal approach. There were 11 males and 13 females with age ranging from 21 to 63 years (average, 39.5). There were 3 patients in $\mathrm{T}_{4 / 5}, 2$ patients in $\mathrm{T}_{5 / 6}, 3$ patients in $\mathrm{T}_{7 / 8}, 3$ patients in $\mathrm{T}_{8 / 9}, 4$ patients in $\mathrm{T}_{9 / 10}, 3$ patients in $\mathrm{T}_{10 / 11}$, and 6 patients in $\mathrm{T}_{11 / 12}$. Patients had different degraded local kyphosis deformity shown on X-ray, and different degraded bone destruction and abscess in thoracic spine shown on CT and MRI before the operation. All of the patients before the regular anti tuberculosis treatment for 2 to 4 weeks, the surgical approach used by paraspinal muscle approach, postoperative regular anti tuberculosis treatment for 9 to 12 weeks. Record the clinical symptoms of patients before and after surgery, preoperative Frankel functional classification of spinal cord injury, the operative time, intraoperative blood loss, postoperative ESR, CRP, complications, VAS score, ODI score and Cobb angle changes, imaging check regularly to evaluate the fusion and follow-up of nerve functional recovery. Results: The average operation time was $198 \mathrm{~min}$. The average blood loss was $436 \mathrm{ml}$. There were no severe complications during and after operation. All patients were followed up for 1 year to 2 years, average 1.5 years of follow-up, the clinical symptoms improved significantly after operation and last follow-up ESR, CRP, VAS score, ODI score and Cobb angle were significantly improved after operation $(\mathrm{P}<0.05)$, grade I Eck fusion, the fusion rate was $100 \%$ and the neurological function were improved. Conclusion: on the basis of strict anti tuberculosis chemotherapy, the use of paraspinal muscle gap approach for the treatment of thoracic tuberculosis is less invasive, less destructive to spinal stability, and can achieve obvious curative effect. It is worthy of clinical application.
\end{abstract}




\section{Keywords}

Thoracic Vertebrae, Tuberculosis, Spinal, Thoracic Paraspinal Approach

\section{Introduction}

Spine is a common site of extrapulmonary tuberculosis, about $10 \%$ of tuberculosis patients with bone and joint tuberculosis, the part of the patients involved in about $50 \%$ of the spine [1]. Adult spine tuberculosis mainly to the edge of tuberculosis is more common, because the thoracic spinal canal volume is small, spinal canal reserve gap is small (transverse diameter than the width of $6 \mathrm{~mm}$, sagittal diameter than the spinal cord length of only less than $2 \mathrm{~mm}$ ), and thoracic blood supply Poor [2], due to the thoracolumbar anatomical structure of the particularity and complexity of spinal tuberculosis often occur in the thoracolumbar, lesions mainly involved in the spine before the column. Spinal tuberculosis to vertebral destruction, paraspinal abscess formation, kyphosis and compression of the spinal cord, and nerve is characterized. Spinal tuberculosis treatment can take non-surgical treatment and surgical treatment. Non-surgical treatment of spinal tuberculosis is the main treatment, commonly used in the early stage of spinal tuberculosis, poisoning symptoms of light, no obvious bone destruction, sequestrum, empty or abscess formation, the patients are sensitive to tuberculosis drugs, or patients are generally worse than recent surgery [3]. Most patients can be cured by strict, standardized non-surgical treatment. But some non-surgical treatment is invalid, with neurological dysfunction or spinal deformity that needs surgery. The purpose of spinal tuberculosis surgery is to completely remove the local lesions, restore nerve function, reconstruction of spinal stability and normal sequence [4] [5] [6] [7]. Spinal tuberculosis surgery is more generally divided into anterior, posterior, before and after the joint approach, the choice based on the lesion involving the site, the scope of vertebral destruction, kyphosis and patients in general to conduct a comprehensive assessment [8] [9]. In recent years, the literature reported by the paravertebral space approach (Wiltse gap approach) in the treatment of thoracolumbar fractures, lumbar intervertebral protrusion and lumbar spondylolisthesis compared to the traditional posterior median approach to retain a large part of the split muscle, Column stability, reducing the incidence of postoperative low back pain, which has been widely used. In this study, 24 cases of thoracic tuberculosis patients were retrospectively analyzed by using the minimally invasive paravertebral muscle gap in our hospital from March 2011 to March 2016, and the intervertebral fusion was performed. Satisfactory results are reported below.

\section{Materials and Methods}

\subsection{General Information}

This is a retrospective study for analytical purposes over 5 years (June 2011 to 
August 2016). 24 patients with mono-segmental thoracic spine tuberculosis were treated by transfacet debridement combined with bone grafting and internal fixation through paraspinal approach. There were 11 males and 13 females with age ranging from 21 to 63 years (average, 39.5). all single stage vertebral involvement patients, including 3 cases of $\mathrm{T}_{4 / 5}, 2$ cases $\mathrm{T}_{5 / 6}, 3$ cases $\mathrm{T}_{7 / 8}, 3$ cases $\mathrm{T}_{8 / 9}, 4$ cases $\mathrm{T}_{9 / 10}, 3$ cases $\mathrm{T}_{10 / 11}, 6$ cases of $\mathrm{T}_{11 / 12}$. The patients mainly manifested as chest pain, dead bone abscess and limited in scope. The visual analog scale (visual, analogue scale, VAS) score was $8.6 \pm 1.5$, Oswestry disability index (Oswe Stry disability index, ODI) is $79 \pm 5$. All of the patients had different degree of spine kyphosis, before and after the convex Cobb angle of $17^{\circ}-41^{\circ}$, the average $\left(30.1^{\circ}-5.6^{\circ}\right)$, $\mathrm{X}$-ray, CT and MRI showed the destruction of all lesions had different degrees of bone formation, vertebral collapse and abscess surgery. The Frankel functional classification of spinal cord injury, including 3 cases of grade B, 9 cases of grade C, 8 cases of grade D, 4 cases of grade E. According to the patient's medical history, clinical manifestations and signs, imaging data (X-ray, CT, MRI) and experiment Room examination (TB chip test and T-Spot test) for primary clinical diagnosis. Intraoperative specimens (pus, granulation tissue, etc.) were confirmed by pathological examination.

\subsection{Sample Selection Criteria}

Inclusion criteria: 1) diagnosis of thoracic tuberculosis. 2) progressive or active spinal tuberculosis with spinal cord injury. 3) tuberculosis involving a movement segment, vertebral body confined to the side and a high degree of loss of not more than $1 / 2$ Vertebral body. 4) paralysis cold abscess confined in the destruction of the vertebral periphery, spinal canal abscess located in the side of the spinal canal. 5) Frankel spinal cord injury function graded $C$ grade and above.

Exclusion criteria: 1) non-tuberculous spinal infection, cancer, trauma and other diseases. 2) lesion damage range (lesion range $>1$ vertebral height), the posterior can not carry out a thorough removal of lesions. 3 ) multi-segment (>2 spinal motility units) or jumping spine tuberculosis. 4) the existence of previous thoracolumbar surgery history, accompanied by congenital spinal deformities, ankylosing spondylitis and spinal tumors and other historical history. 5) there are multiple segments of the huge paraspinal abscess, or flow abscess. 6) patients can not tolerate surgery Severe cardiopulmonary dysfunction.

\subsection{Preoperative Preparation}

Detailed history data collection, improve preoperative laboratory tests and imaging studies, parallel Frankel nerve function assessment. All patients were treated with normal anti-tuberculosis drugs before surgery (quadruple therapy: isoniazid $0.3 \mathrm{~g} / \mathrm{d}$, rifampicin, $0.45 \mathrm{~g} / \mathrm{d}$, streptomycin $0.75 \mathrm{~g} / \mathrm{d}$ and ethambutol $0.75 \mathrm{~g} / \mathrm{d}, 2$ - 4 weeks), while giving liver and nutritional support treatment, regular review of blood, liver and kidney function and ESR, CRP, to be ESR $<50$ $\mathrm{mm} / \mathrm{h}$ or $\mathrm{CRP}<30 \mathrm{mg} / \mathrm{L}$ after elective surgery. 


\subsection{Surgical Methods}

Patients with preoperative catheterization catheterization, tracheal intubation after the success of general anesthesia turn prone position, $\mathrm{C}$-arm perspective to determine the lesion segment, do mark. Routine disinfection of the towel to the vertebral body as the center, take the middle of the incision, along the middle of the shallow fascia and shallow chest and back muscles and pull to both sides, revealing deep muscle and sarcolemma, along the spinous process. On both sides of the side of the opening 1 transverse cut myometrium, along the adipose tissue to find the muscle gap, from the inside of the more split muscle, lateral long muscle gap blunt separation, can reveal the facet of the facet and the transverse process, upper and lower segments of the vertebral implantation pedicle screw, contralateral pre-bending titanium rod temporary fixation, to avoid inter-segmental displacement lead to spinal cord injury (at least 2/3 of the vertebral body and the pedicle are not damaged), the proximal screw as close as possible to the vertebral end plate, distal screw in the pedicle into the bottom, as far as possible away from the End plate or choose shorter pedicle screw. If one or both sides of the vertebral body and pedicle damage is serious, the lesion vertebral no staple, the fixed section of the corresponding extension (approximately 4 $\mathrm{cm}$ ), resection of the lateral articular facet joint, partial pedicle resection and careful removal of the periosteum together with the wall (the lesion involving the intervertebral space) Pleural effusion and bone knife to remove the lesion hardening bone and granulation tissue to have bleeding so far. To normal saline, Hydrogen peroxide and diluted isoniazid solution to a large number of repeated washing lesions and sinus after the conventional hemostasis. Lesion area into the streptomycin $1 \mathrm{~g}$ and isoniazid $0.2 \mathrm{~g}$, and take the body iliac bone (defect can be used when the larger titanium mesh plus autogenous bone support bone graft), and streptomycin powder stir after intercourse Line bone graft fusion, contralateral lesions also used to deal with the above method. Pre-bent bilateral titanium rods to normal curvature, bilateral titanium rods and correction of kyphosis, bilateral titanium rods compression locked. If necessary, to connect the horizontal link to maintain stability, respectively, on the left and right sides of the placement of a number of Pan's tube, one by one to close the incision, the surgical removal of lesions sent to pathological examination and bacterial culture (Figure 1).

\subsection{Postoperative Treatment}

Postoperative anti-infective treatment for 3 to 5 days and with the blood circulation pain, nutritional nerve and other symptomatic support treatment, conventional surgical incision dressing, 2 to 3 days after the drainage tube $(24 \mathrm{H}$ drainage less than $50 \mathrm{ml}$ ), if the cerebrospinal fluid leakage Indwelling drainage tube 1 week after the pull out, the patient 1 week after wearing a brace to get out of bed activities, 6 months after the removal of bone graft removal. Postoperative Regular anti-tuberculosis treatment for 18 months, while the liver treatment. Regular monitoring of ESR, CRP and liver and kidney function, postoperative 3, 


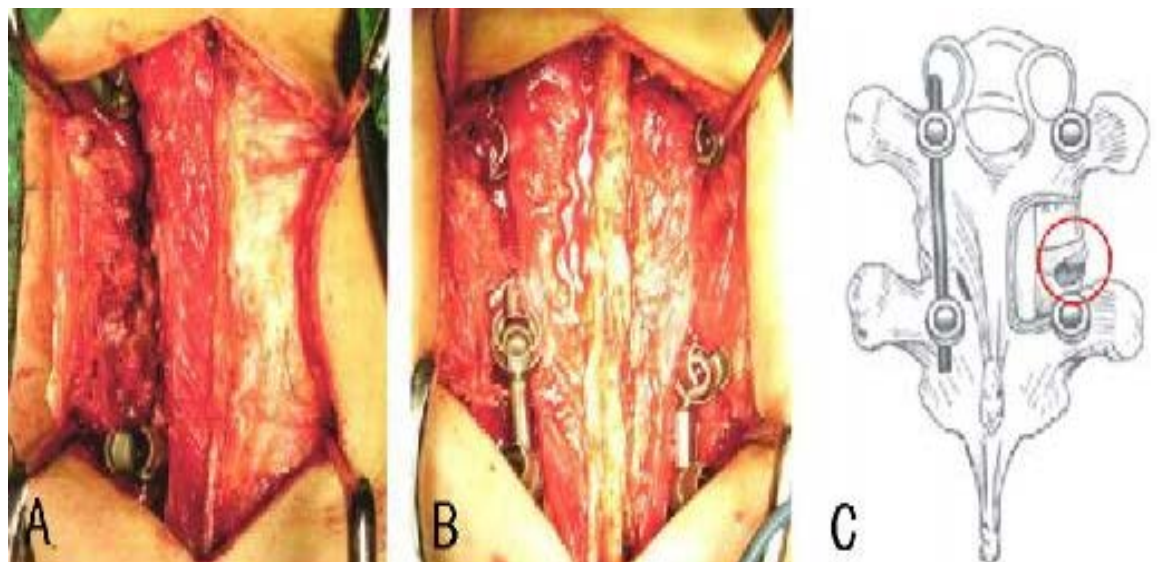

Figure 1. Schematic diagram of surgery (drawing by Jinpeng, Zheng) in the operation of bilateral segmental pedicle screw, decompression in the side contralateral to the installation of connecting rod and temporary fixation with transarticular approach when the small joint excision range, circles represent the intervertebral space to be processed, the inside is the spinal cord. If the lesion is large show view, caudal pedicle can be removed, to expand our horizons.

$6,12,18,24$ months to review X-ray or CT, the necessary time feasible MRI examination.

\subsection{Observation of Efficacy}

1) Intraoperative and postoperative complications assessment: recorded the operation time, bleeding volume, postoperative complications.

2) Clinical efficacy evaluation: a) the postoperative follow-up period VAS pain score and Oswestry disability index (ODI), to evaluate the degree of pain and improve functional recovery. $b$ ) The measurement of preoperative, postoperative and final follow-up Cobb angle, Cobb angle change assessment by correcting the degree of kyphosis. c) The use of Frankel classification method to evaluate spinal nerve damage. To evaluate preoperative and last follow-up respectively.

3) Imaging assessment the bone fusion was evaluated using the Eck fusion classification criteria [8]. Grade II: may be fusion, bone graft, the lower edge of the trabecular bone, no gaps, bone graft is not completely remodeling. Grade III: the level of bone marrow, may not be fused, bone on the lower edge of the trabecular bone without bridging and the emergence of gaps. Grade IV: due to bone graft absorption or collapse is not fully integrated. Grade V: can not be evaluated.

\section{Statistical Processing}

All the indicators in this study were observed by the same group of physicians and statistical data, were recorded SPSS 21.0 statistical software for processing. The quantitative data involved, before and after surgery, were used paired $t$ test; qualitative data comparison using chi-square test, set $\mathrm{P}<0.05$ for the difference was statistically significant. 


\section{The Results}

\subsection{Intraoperative Observation}

In 24 cases, the operation was completed successfully. The operation time was $180-240 \mathrm{~min}$, the average was $198 \mathrm{~min}$. The total blood loss was $300-600 \mathrm{ml}$, and the average blood loss was $436 \mathrm{ml}$ (Table 1).

\subsection{Complications and Postoperative Wound Healing after Surgery}

Wound dehiscence induced delayed healing in 3 cases after surgery, 2 patients with superficial incision infection, after debridement and vacuum suction after suturing again, two were healed. There were 1 cases of cerebrospinal fluid in patients after 1 week after pulling out the drainage tube and drainage tube indwelling suture incision, after 3 days of recovery.

\subsection{Postoperative Follow-Up}

All the patients were followed up; the follow-up time was 1 - 2 years, with an average of 1.5 years, no internal fixation loosening and fracture during the follow-up period, no spinal instability, no sinus formation, no recurrence of tuberculosis.

1) ESR and C-reactive protein index improvement: Patients received treatment of $2-4$ weeks of regular anti tuberculosis, postoperative follow-up, ESR, CRP respectively from preoperative $(40.7 \pm 10.4) \mathrm{mm} / \mathrm{h}(15.7 \pm 4.8) \mathrm{mg} / \mathrm{L}$ decreased after operation $(28.8 \pm 7.1) \mathrm{mm} / \mathrm{h}(11.3 \pm 2.4) \mathrm{mg} / \mathrm{L}$, the statistical analysis, the difference has statistical significance $(P<0.05)$, at last follow-up, ESR, CRP is significantly decreased after operation $(\mathrm{P}<0.05)$ (Table 2$)$.

2) Cobb corner correction: All of the patients before and after operation of convex Cobb angle of $17^{\circ}-41^{\circ}$, the average $\left(30.1^{\circ} \pm 5.6^{\circ}\right)$, postoperative improvement to $8^{\circ}-20^{\circ}$, the average $\left(14.6^{\circ} \pm 3.5^{\circ}\right)$, during the last follow-up, the Cobb angle of $10^{\circ}-23^{\circ}$, the average $\left(15.9^{\circ} \pm 4.2^{\circ}\right)$ before and after operation. Contrast, at the end of the follow-up and postoperative contrast showed no significant difference $(\mathrm{P}>0.05)$, after operation and last follow-up when compared with the preoperative, the difference was statistically significant $(\mathrm{P}<0.05)$ (Table 3).

Table 1. The operation time and the amount of bleeding in different operation segments.

\begin{tabular}{cccc}
\hline Segment & Case & Time $(\mathrm{min})$ & Intraoperative blood loss $(\mathrm{ml})$ \\
\hline $\mathrm{T}_{4 / 5}$ & 3 & $195 \pm 43$ & $412 \pm 81$ \\
$\mathrm{~T}_{5 / 6}$ & 2 & $215 \pm 55$ & $419 \pm 76$ \\
$\mathrm{~T}_{7 / 8}$ & 3 & $203 \pm 47$ & $413 \pm 79$ \\
$\mathrm{~T}_{8 / 9}$ & 3 & $196 \pm 32$ & $450 \pm 87$ \\
$\mathrm{~T}_{9 / 10}$ & 4 & $209 \pm 33$ & $508 \pm 80$ \\
$\mathrm{~T}_{10 / 11}$ & 3 & $205 \pm 38$ & $410 \pm 75$ \\
$\mathrm{~T}_{10 / 11}$ & 6 & $185 \pm 42$ & $400 \pm 82$
\end{tabular}


Table 2. Changes of ESR and CRP before and after operation.

\begin{tabular}{cccc}
\hline Time & Case & ESR $(\mathrm{mm} / \mathrm{h})$ & CRP $(\mathrm{mg} / \mathrm{L})$ \\
\hline Preoperative & 24 & $40.7 \pm 10.4$ & $15.7 \pm 4.8$ \\
Postoperative 7d & 24 & $28.8 \pm 7.1$ & $11.3 \pm 2.4$ \\
Final follow-up & 24 & $10.1 \pm 2.0$ & $1.8 \pm 1.2$ \\
\hline
\end{tabular}

Note: postoperative ESR and CRP values were significantly lower than those before operation, and the difference was statistically significant $(\mathrm{P}<0.05)$.

Table 3. The changes of mean Cobb angle at preoperative, postoperative and final follow-up.

\begin{tabular}{cccc}
\hline Project & Preoperative & Postoperative $7 \mathrm{~d}$ & Final follow-up \\
\hline Cobb angle & $30.1^{\circ} \pm 5.6^{\circ}$ & $14.6^{\circ} \pm 3.5^{\circ}$ & $15.9^{\circ} \pm 4.2^{\circ}$ \\
\hline
\end{tabular}

Note: before operation, after vs, $\mathrm{t}=11.50, \mathrm{P}=0.00$; Vs before operation at the end of the follow-up, $\mathrm{t}=9.94$, $\mathrm{P}=0.00$; VS after the last follow-up, $\mathrm{t}=1.16, \mathrm{P}=0.25$.

3) VAS score and ODI index improvement: VAS, ODI scores of the patients in the whole group at the last 7, D and last follow-up were statistically significant $(\mathrm{P}<0.05)$ (Table 4).

4) According to the imaging findings and Eck fusion grading criteria, there were no internal fixation loosening, breakage and displacement after X-ray examination at the last follow-up visit. The CT showed good bone graft fusion, and the Eck fusion grade was grade I, and the fusion rate was 100\% (Figure 2).

5) Recovery of neurological function: Preoperative $B$ grade 3 cases, restore to $C$ grade 1 cases, $D$ class 1 cases, $E$ class 1 cases, $C$ class 9 cases, restore to D grade 2 cases, $\mathrm{E}$ class 7 cases, D class 8 cases all recovered to $\mathrm{E}$ grade. Before and after the operation contrast, the difference was statistically significant $(P<0.05)$ (Table 5).

\section{Discuss}

The purpose of spinal tuberculosis surgery is to: completely remove the lesion, relieve nerve compression, reconstruction of spinal stability, correction of spinal deformity [10]. Thoracic vertebral space because of its smaller, resulting in little buffer space, more likely to lead to high paraplegia and other adverse consequences. Thoracic tuberculosis surgery a variety of methods [11] [12] [13] [14], but each has its own advantages and disadvantages, the clinical practice is not yet recognized the best surgical method.

Paravertebral space approach to the generation: Spinal parietal muscle is divided into cleft muscle, long muscle and iliac muscle, which is the trunk muscle is an important muscle, mainly the role of stable spine, cleft muscle from the upper vertebral spinous process, Oblique walking to the lower vertebral body of the transverse process, can be divided into the surface of the muscle bundle and deep muscle bundle, the surface of the muscle bundle can span multiple vertebral bodies, with directional specificity, the role of vertebral activity, deep 
Table 4. The VAS score and ODI score were scored before, 1 week after operation and at the last follow-up.

\begin{tabular}{cccc}
\hline Idex & Case & VAS & ODI \\
\hline Preoperative & 24 & $8.6 \pm 1.5$ & $79 \pm 5$ \\
Postoperative 7d & 24 & $2.7 \pm 1.3^{\mathrm{a}}$ & $26 \pm 4^{\mathrm{a}}$ \\
Final follow-up & 24 & $1.4 \pm 1.3^{\mathrm{ab}}$ & $19 \pm 6^{\mathrm{ab}}$ \\
$\mathrm{t}$ & & 243.210 & 1299.987 \\
$\mathrm{P}$ & & 0.00 & 0.00 \\
\hline
\end{tabular}

Note: VAS was the visual analogue scale, and ODI was the Oswestry dysfunction index. A was compared with preoperative ${ }^{\mathrm{b}} \mathrm{P}<0.01$, compared with the postoperative $7 \mathrm{~d}$, ${ }^{\mathrm{b}} \mathrm{P}<0.01$.

Table 5. The grading of Frankelspinal cord injury before operation and at the end of follow-up.

\begin{tabular}{ccccccc}
\hline Time & Case & A degree & B degree & C degree & D degree & E degree \\
\hline Preoperative & 24 & 0 & $3^{*}$ & $9^{*}$ & $8^{*}$ & $4^{*}$ \\
Final follow-up & 24 & 0 & 0 & 1 & 3 & 20 \\
\hline
\end{tabular}

Note: ${ }^{*}$ compared with last follow-up, $\mathrm{P}<0.05$.

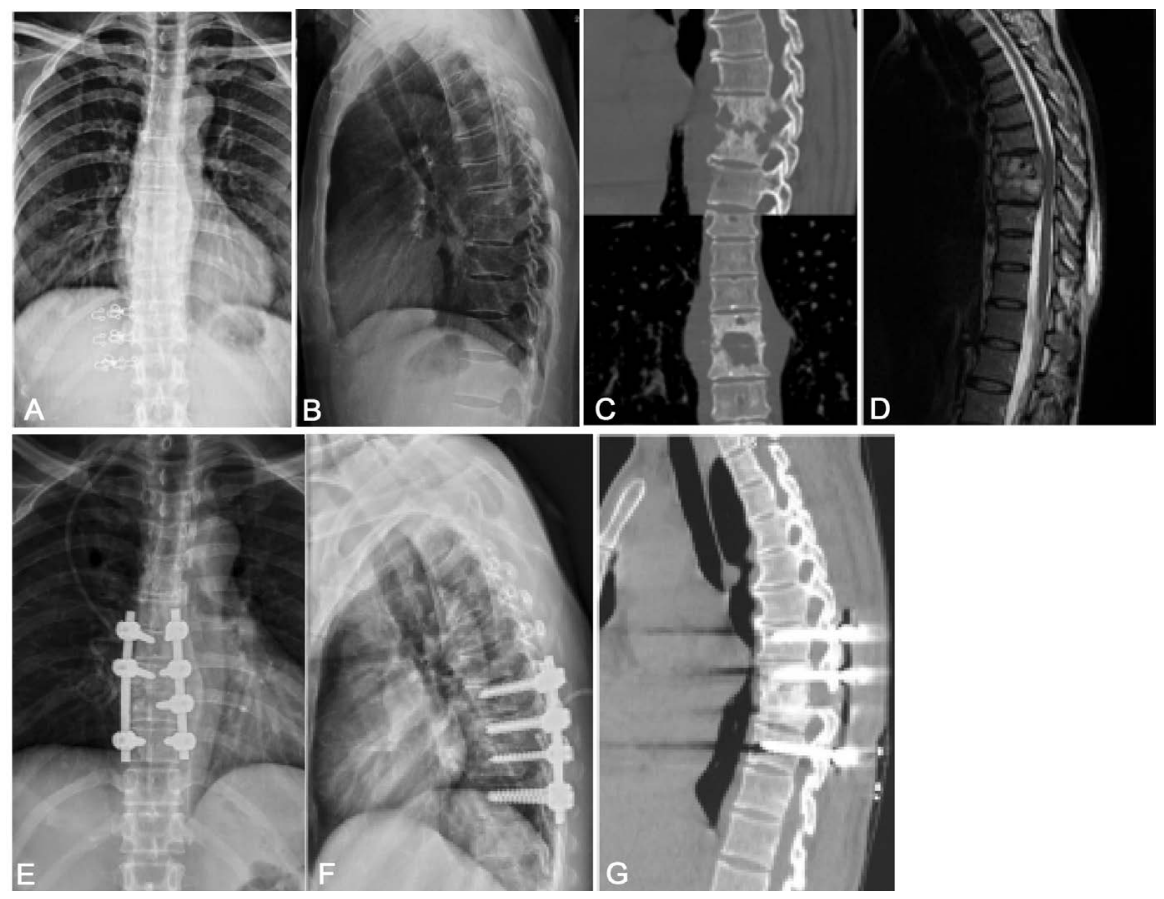

Figure 2. Female, 52y, Back pain for 5 months, The paraspinal muscle gap through costotransversectomy debridement, interbody bone graft fusion and internal fixation for the treatment of $T_{9}$, the right vertebral destruction, $T_{7 \sim 10}$ and $T_{9}$ on the left side of the screw bilateral A, B: preoperative lateral radiograph $\mathrm{T}_{8 / 9}$ intervertebral space collapse damage. $\mathrm{C}$ : preoperative sagittal plane CT, coronal slices showed $\mathrm{T}_{8 / 9}$ vertebral lesions, paravertebral abscess formation, $\mathrm{T}_{8 / 9}$ bone destruction, which $\mathrm{T}_{9}$ on the right side of the most serious damage. $\mathrm{D}$ : preoperative sagittal $\mathrm{T}_{2}$ weighted MRI showed $\mathrm{T}_{8 / 9}$ vertebral lesions, vertebral disc destruction, signal change and collapse, the dural sac mild compression, ventral CSF. E, F: lost; after 7d X-ray showed good position of internal fixation. G: 1 year after operation of thoracic CT+ reconstruction showed tuberculous lesions can control (no paravertebral abscess), $\mathrm{T}_{8 / 9}$ bone graft has full integration. 
muscle bundle is only two Adjacent vertebrae, the spinal segment between the balance of movement and shear force from the control [15]. Paravertebral muscle approach by Wiltse et al. [16] in 1968 proposed in the lumbar spine and the longest muscle between the physiological gap lumbar posterior lumbar surgery, it is also known as Wiltse gap approach. Because the approach does not need to divest the paraspinal muscles, with tissue damage is small, exposure and stapling operation is convenient, lower complications and other advantages, and to avoid the paraspinal myelopathy and long-term traction caused by the source Sexual damage, reducing the incidence of postoperative low back pain [17] [18]. In recent years, scholars at home and abroad through the extensive application of paravertebral muscle gap approach (Wiltse gap approach) surgery for thoracolumbar vertebral fracture, lumbar spondylolisthesis and lumbar degenerative disease. In the spine of the thoracic also exist the same muscle gap, we use the gap approach can be better to reveal the transverse process, small joints and lamina, set nail convenience, and by removing the lateral articular facet joint and part of the lamina (Figure 1), if necessary, can be removed lesions vertebral pedicle (through the pedicle) revealed vertebral space, paraspinal and spinal canal lesions, the full removal of lesions and bone graft. Xu Yong et al. [19] studies have shown that the use of paravertebral space approach for the treatment of thoracic tuberculosis.

Paravertebral muscle gap indications: The fusion of internal and external fixation of bone graft is widely used in the treatment of lumbar disc herniation, lumbar spondylolisthesis and thoracolumbar spine fracture, but it is used in the treatment of thoracolumbar tuberculosis. Less, there is also large controversy. In addition to posterior surgery to further damage the stability of the spine and increase the possibility of tuberculosis lesions contaminated rear structure, the focus of the controversy is the thoracic and lumbar spine tuberculosis to take the technical treatment of surgical indications is not clear. Although the current approach to the treatment of thoracolumbar tuberculosis is not clear, but the basic principle is to do not increase the trauma and risk under the premise of choice, the choice of the simpler and more feasible method achieves the goal of effective treatment. According to this group of patients and related literature review, for the diagnosis of the need to take the paravertebral muscle gap through the articular process of thoracolumbar spine tuberculosis treatment indications: a) thoracolumbar spine tuberculosis, the disease involving a single movement segment; Vertebral involvement is limited to one side and a high degree of loss does not exceed 1/2 vertebral body. b) Paralysis cold abscess confined to the destruction of the vertebral periphery, spinal canal abscess located in the spinal canal side, can directly reach the complete clearance and nerve Decompression. c) Progressive or active spinal tuberculosis with spinal cord injury. d) Frankel spinal cord injury function graded $\mathrm{C}$ grade and above. Therefore, the use of paravertebral muscle gap approach for the treatment of thoracolumbar tuberculosis, the preoperative not only to fully assess the patient's general situation, need to care- 
fully read the patient's spinal X-ray, CT and MRI to clear the lesion responsibility segment, Nerve compression and kyphosis, etc. if necessary, please see the Department of imaging examination films to avoid preoperative errors caused by surgical difficulties, or the need to change the surgical approach after the middle of the way, before or after the joint approach to achieve Therapeutic purpose.

3) Paraspinal muscle gap approach advantages

According to the indications of the operation, careful examination by surgery, take the paravertebral muscle gap approach to complete the lesion removal, bone fusion and spinal sequence, the stability of reconstruction. This study shows that, compared with the traditional posterior approach, has the following advantage: a) by the paravertebral muscle gap approach, to avoid the paravertebral split muscle stripping, to maximize the protection of the deep cleavage of the nerve, Reducing the multi-split muscle degeneration of the region, reducing the thoracolumbar "axial pain" the incidence of the maximum to avoid the occurrence of iatrogenic injury. b) to retain the spinous process, interspinous and spine on the ligament and other most of the posterior column structure, to reduce the normal physiological structure of paravertebral muscle damage, so that the overall stability of the spine better to ensure a good "internal brake" effect, Lesion control and bone fusion. c) Posterior approach to the posterior approach can be more accurate and accurate access to the pedicle screw implantation point, so that surgery is simpler, shorter operation time, less bleeding. d) Relative to the traditional posterior approach, the paraspinal muscle gap to retain the paraspinal muscles to reduce the median approach to the lamina and transverse process of paravertebral muscle injury, significantly reduced the surgical incision delayed healing, infection and abscess Formation and other complications. e) Through the joint approach advantages. Not only can be more fully clear the intervertebral necrotic tissue, sequestrum, paraspinal abscess and other spinal canal lesions, and can look directly into the spinal cord spinal cord, the spinal canal pressure on the full decompression, lifting the spinal cord compression. And can be better between the vertebral autologous bone graft, restore sagittal curvature of the spine, to prevent long-term kyphosis occurred. Some scholars have reported that by the paravertebral gap approach not only can not completely remove the abscess, but also cause tuberculosis recurrence and spread, which makes the paravertebral muscle gap approach to the surgical application is limited, but our research results show that: On the one hand, the paravertebral muscle gap approach is the epidural operation, will not Mycobacterium tuberculosis into the spinal cord, causing tuberculous cerebrospinal meningitis and other complications; the other hand, even if the "thorough" lesions [20] study that anti-tuberculosis drugs in patients with spinal tuberculosis disease has a significant difference in the distribution of tissue: in the hardening of the sub-normal bone up to the effective sterilization concentration, in the Hardened wall is low concentration. Through the follow-up of patients, no recurrence of tuberculosis recurrence cases, studies have shown that the paravertebral muscle 
gap surgery can remove the lesion to the normal bone, combined with normal chemotherapy, can be relatively small in the case of relatively complete clearance Lesions. In short, for the limitations of the scope of the thoracic tuberculosis patients, the use of paravertebral muscle gap approach, not only the surgical trauma is small, easy to operate, less complications, the greatest degree of stability to maintain the spine, reduce postoperative refractory chest and back pain occur.

The limitations of this study: We through the above case study found that the paravertebral space approach does not apply to the following thoracic tuberculosis patients: a) multiple vertebral segments involved. b) For the larger side of the abscess, the intervertebral muscle gap approach may not be able to clear it completely, still need to use the road before the thorough removal of lesions. c) For the spinal canal lesions involving a large range or for Frankel spinal cord injury function grading for the A, B level of the patient, the need for larger surgical operation space to completely remove the lamina or even a wider range of spinal decompression, vertebral Bypass muscle gap approach surgery is extremely difficult, spinal canal decompression is not complete. Therefore, the subjects selected in this study have some limitations compared with those of the study subjects selected by the previous or posterior or posterior approach. Second, because the study did not set up the corresponding control group of patients, but through retrospective literature review of surgical efficacy comparison, the results of a certain degree of subjective bias. In addition, this study is a retrospective case analysis, the number of cases is small, the follow-up time is not long and other shortcomings, but also the needs for multi-center research results to further verify the rationality of the operation.

To sum up, on the basis of standardized anti-tuberculosis treatment of drugs, the paravertebral muscle gap approach to surgery can completely remove the lesions, bone fusion and reconstruction of the normal spine sequence and stability, and the operation not only has Simple operation, less bleeding, shorter operative time, less complication and higher safety, and achieved the same clinical effect as the traditional posterior approach. It provides an effective method for the treatment of thoracic tuberculosis; advocate the idea of minimally invasive spine surgery.

\section{Consent}

We received written informed consent from the patient for publication of this article. This study was approved by the local ethics committee and all the authors contributed to the writing of this article.

\section{Conflicts of Interest}

No conflicts of interest were declared by the authors.

\section{References}

[1] McLain, R.F. and Isada, C. (2004) Spinal Tuberculosis Deserves a Place on the Radar Screen. Cleveland Clinic Journal of Medicine, 71, 537-549. 
https://doi.org/10.3949/ccjm.71.7.537

[2] Huan, Y.W., Wang, Q.P., Dai, X.W., et al. (1998) National Thoracic Spinal Cord and Spinal Canal Observation and Measurement. Journal of Cervical and Lumbago, 19, 173-175.

[3] Zhang, Z.H., Li, L.T., Luo, F., et al. (2014) The Early Diagnosis of Mild Spinal Tuberculosis and Outcomes of Nonoperative Treatment. Chinese Journal of Orthopaedic Trauma, 34, 177-182.

[4] Soares do Brito, J., Batista, N., Tirado, A., et al. (2013) Surgical Treatment of Spinal Tuberculosis: An Orthopedic Service Experience. Acta Medica Portuguesa, 26, 349356.

[5] Moon, M.S. (2014) Tuberculosis of Spine: Current Views in Diagnosis and Management. Asian Spine Journal, 8, 97-111.

[6] Jutte, P.C. and van Loenhout-Rooyackers, J.H. (2006) Routine Surgery in Addition to Chemotherapy for Treating Spinal Tuberculosis. The Cochrane Database of Systematic Reviews, 5, Article ID: CD004532. https://doi.org/10.1002/14651858.CD004532.pub2

[7] Issack, P.S. and Boachie-Adjei, O. (2012) Surgical Correction of Kyphotic Deformity in Spinal Tuberculosis. International Orthopaedics, 36, 353-357. https://doi.org/10.1007/s00264-011-1292-9

[8] Meena, S., Mittal, S. and Chowdhary, B. (2014) Spinal Tuberculosis: Which Is the Best Surgical Approach? Medical Principles and Practice, 23, 96. https://doi.org/10.1159/000353146

[9] Eck, K.R., Lenke, L.G., Bridwell, K.H., et al. (2000) Radiographic Assessment of Anterior Titanium Mesh Cages. Journal of Spinal Disorders, 13, 501-510.

[10] Mehta, J.S. and Bhojraj, S.Y. (2001) Tuberculosis of the Thoracic Spine. A Classi-Fication Based on the Selection of Surgical Strategies. The Journal of Bone and Joint Surgery. British Volume, 83, 859-863. https://doi.org/10.1302/0301-620X.83B6.11142

[11] Jain, A.K. and Jain, S. (2012) Instrumented Stabilization in Spinal Tuberculosis. International Orthopaedics, 36, 285-292. https://doi.org/10.1007/s00264-011-1296-5

[12] Cui, X., Li, L.T. and Ma, Y.Z. (2016) Anterior and Posterior Instrumentation with Different Debridement and Grafting Procedures for Multi-Level Contiguous Thoracic Spinal Tuberculosis. Orthopaedic Surgery, 8, 454-461. https://doi.org/10.1111/os.12288

[13] Ahsan, K. and Sakeb, N. (2016) Single-Stage Posterior Instrumentation for Progressive Tubercular Thoracic and Thoracolumbar Kyphosis. Journal of Orthopaedic Surgery (Hong Kong), 24, 344-349. https://doi.org/10.1177/1602400314

[14] Shi, J., Yue, X., Niu, N., et al. (2016) Application of a Modified Thoracoabdominal Approach That Avoids Cutting Open the Costal Portion of Diaphragm during Anterior Thoracolumbar Spine Surgery. European Spine Journal, Epub Ahead of Print.

[15] Fan, S.W., Hu, Z.J., Qian, Q., et al. (2009) Comparative Study of Posterior Lumbar Interbody Fusion with Small Incision and Conventional Open Surgery. Zhonghua Department of Orthopedics, 29, 1000-1004.

[16] Wiltse, L.L., Bateman, J.G., Hutchinson, R.H., et al. (1968) The Paraspinal Sacrospinalis-Splitting Approach to the Lumbar Spine. The Journal of Bone and Joint Surgery. American Volume, 50, 919-926. https://doi.org/10.2106/00004623-196850050-00004

[17] Street, J.T., Andrew Glennie, R., Dea, N., et al. (2016) A Comparison of the Wiltse 
versus Midline Approaches in Degenerative Conditions of the Lumbar Spine. Journal of Neurosurgery: Spine, 25, 332-338.

https://doi.org/10.3171/2016.2.SPINE151018

[18] Hyun, S.J., Kim, Y.B., Kim, Y.S., et al. (2007) Postoperative Changes in Paraspinal Muscle Volume: Comparison between Paramedian Interfacial and Midline Approaches for Lumbar Fusion. Journal of Korean Medical Science, 22, 646-651. https://doi.org/10.3346/jkms.2007.22.4.646

[19] Xu, Y., Xu, H. and Li, F. (2017) Paraspinal Approach via Facet Interbody Fusion in the Treatment of Thoracic Tuberculosis. Journal of Orthopaedics, 8, 81-84.

[20] Wang, G., Zhao, H., Wei, M.J., et al. (2009) Distribution of Isoniazid in Different Tissues of Spinal Tuberculosis. Jiangsu Medicine, 35, 669. 